\title{
Laser Ultrasonic System for Surface Crack Visualization in Dissimilar Welds of Control Rod Drive Mechanism Assembly of Nuclear Power Plant
}

\author{
Yun-Shil Choi, Hyomi Jeong, and Jung-Ryul Lee \\ Department of Aerospace Engineering and LANL-CBNU Engineering Institute Korea, Chonbuk National University, \\ 664-14 Deokjin-dong, Deokjin-gu, Jeonju, Jeollabuk-do 561-756, Republic of Korea \\ Correspondence should be addressed to Jung-Ryul Lee; leejrr@jbnu.ac.kr
}

Received 25 December 2012; Accepted 4 March 2013; Published 17 March 2014

Academic Editor: Gyuhae Park

Copyright (C) 2014 Yun-Shil Choi et al. This is an open access article distributed under the Creative Commons Attribution License, which permits unrestricted use, distribution, and reproduction in any medium, provided the original work is properly cited.

\begin{abstract}
In this paper, we propose a J-groove dissimilar weld crack visualization system based on ultrasonic propagation imaging (UPI) technology. A full-scale control rod drive mechanism (CRDM) assembly specimen was fabricated to verify the proposed system. An ultrasonic sensor was contacted at one point of the inner surface of the reactor vessel head part of the CRDM assembly. Qswitched laser beams were scanned to generate ultrasonic waves around the weld bead. The localization and sizing of the crack were possible by ultrasonic wave propagation imaging. Furthermore, ultrasonic spectral imaging unveiled frequency components of damage-induced waves, while wavelet-transformed ultrasonic propagation imaging enhanced damage visibility by generating a wave propagation video focused on the frequency component of the damage-induced waves. Dual-directional anomalous wave propagation imaging with adjacent wave subtraction was also developed to enhance the crack visibility regardless of crack orientation and wave propagation direction. In conclusion, the full-scale specimen test demonstrated that the multiple damage visualization tools are very effective in the visualization of J-groove dissimilar weld cracks.
\end{abstract}

\section{Introduction}

Control rod drive mechanism (CRDM) assembly includes a reactor vessel head (RVH) and many penetration nozzles made of carbon steel and alloy 690, respectively, as shown in Figure 1(a). The two dissimilar metal parts, namely, the RVH and penetration nozzle, are coupled with welding as shown in the unit structure of the CRDM assembly in Figure 1(b). The inner surface of the RVH, which is in direct contact with the primary coolant, is covered with cladding to prevent any reaction between the carbon steel ingredient in the RVH and the boric ingredient in the coolant. During a nuclear power plant (NPP) operation period, thermal and pressure loadings are concentrated on the penetration nozzles and dissimilar metal welding, which are comparatively fragile spots. As the operation period of nuclear plants has increased, there has been an increase in the growth of primary water stress corrosion (PWSCC) on the welds of dissimilar metals or penetration nozzles by cyclic stress. As shown in Figure 2(a), these PWSCCs ultimately grow into surface cracks and become the path of primary water leakage. While the boric acid ingredient in the coolant not only accumulates on the outer surface of the reactor vessel as boric acid deposits, but it also creates a cavity by reacting with the carbon steel ingredient of the RVH, as shown in Figure 2(b) [1]. Practically, corrosions of RVH by boric acid deposits have been demonstrated by Davis-Besse 2002 [2], and a leak of primary coolant water through the inner surface cracks was noticed at the Ohi NPP in Japan, 2004 [3].

In order to detect small amounts of PWSCC in the reactor, an inspection needs to be carried out using an automatic inspection system capable of rapid testing and analyzing because the CRDM assembly has minimal human accessibility. Furthermore, even though PWSCCs grow into surface cracks, they are difficult to detect because they are not open cracks and thus not easily distinguishable with the naked eye. If these kinds of damage were neglected, serious accidents could occur such as fracture of penetration nozzles due to the growth of PWSCC around the welds. If the tests take too long to complete, extreme economic loss ensues 


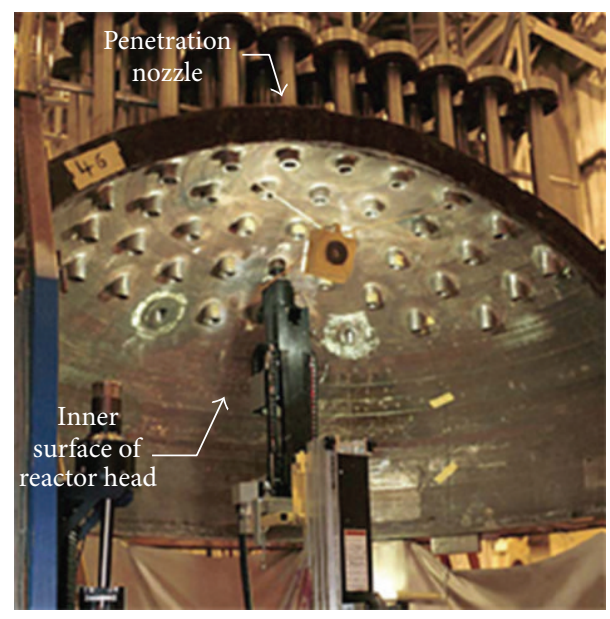

(a)

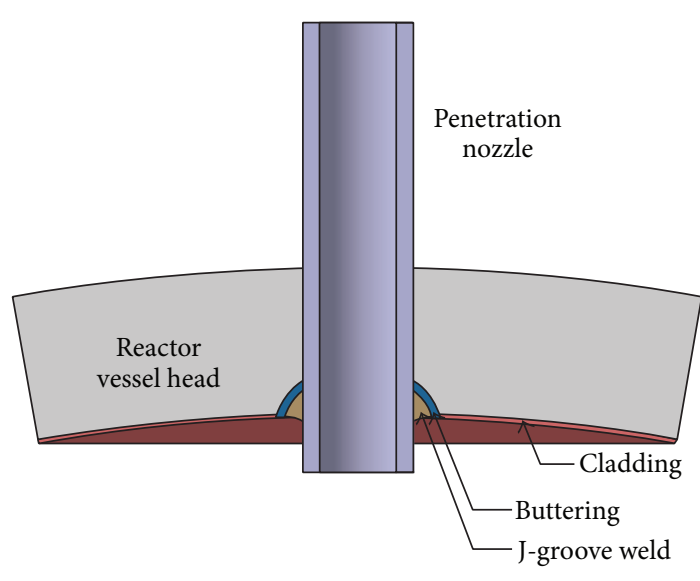

(b)

FIGURE 1: (a) Real photo of control rod drive mechanism (CRDM) assembly [10] and (b) schematic diagram of CRDM assembly.

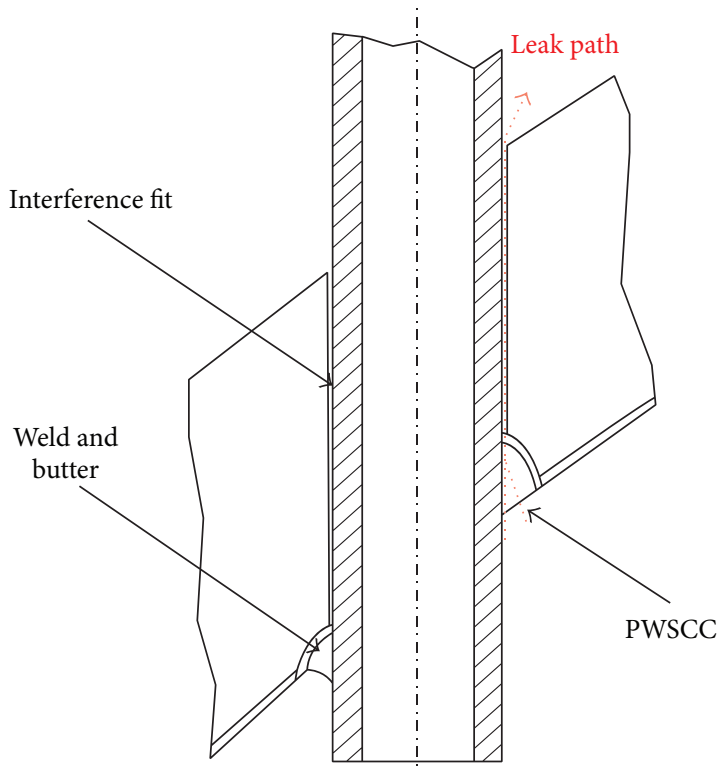

(a)

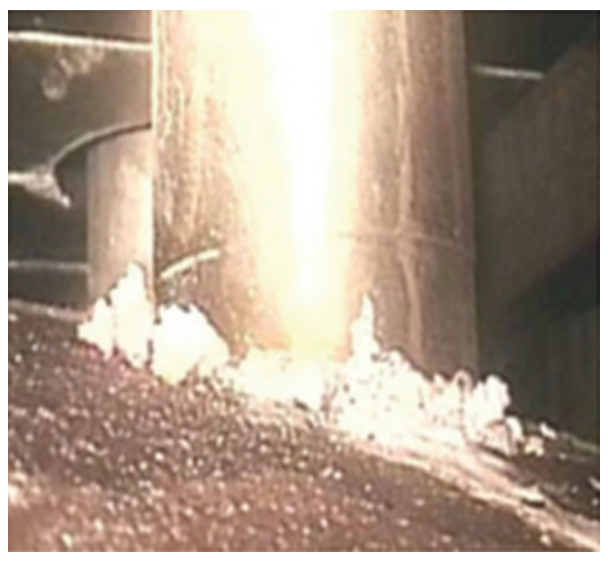

(b)

FIGURE 2: (a) Leak path of primary coolant by PWSCC, (b) Boric acid deposits [1].

because most of the testing then needs to be completed during an out-of-service period.

Various NDT methods are currently being used to detect PWSCC in CRDM assembly such as ultrasonic technique (UT), Eddy current technique (ET), visual technique (VT), and penetrant technique (PT). Cumblidge et al. [4] have presented a PWSCC detecting method based on ET for RVH, J-groove weld, and penetration nozzle, and so forth in a removed-from-service CRDM assembly. However, problems arise from the ET methods such as long inspection time for a small area because of the narrow contact surface of the probe and low reliability caused by a variation of the contacted area between the target surface and the probing area in each probing. For the UT, a PWSCC detecting method using a pulse/echo mode has been presented by Kerr and Alers [5]. The method showed superior penetration depth and serviceability over the ET-based method but it required point-by-point scanning by directly contacting the UT probe. Therefore, fulfilling the testing in a complex welding part and curved RHV is difficult and time consuming. Thus, advanced nondestructive evaluation (ANDE) techniques capable of faster and more reliable scanning should be developed for the CRDM assembly.

In this paper, we design a dissimilar weld crack visualization system for the CRDM assembly. The system can perform noncontact scanning generation of ultrasonic waves on the inner surface of the RVH by impinging the laser pulses Q-switched at $200 \mathrm{~Hz}$. The system can also acquire 


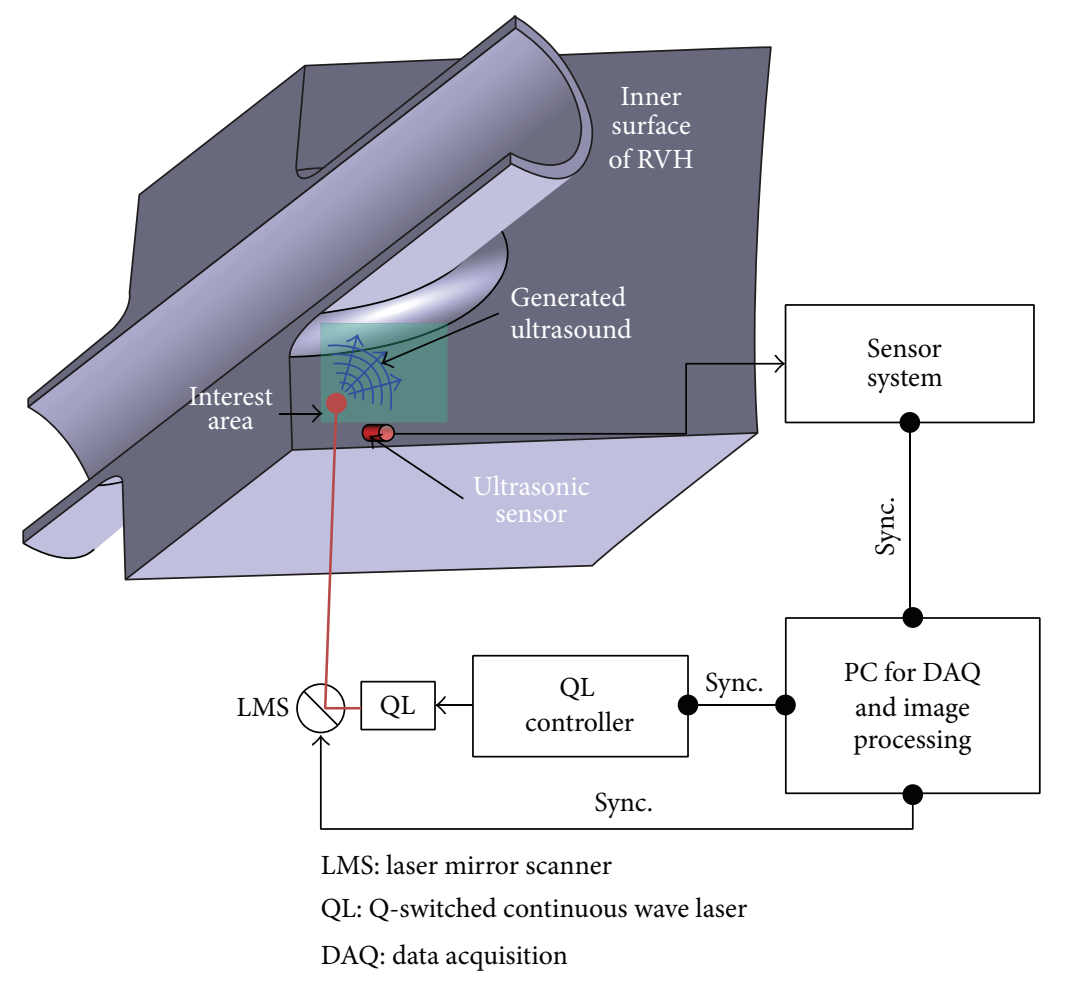

(a)

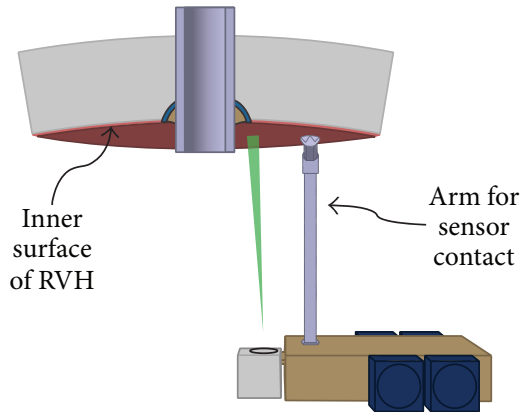

(b)

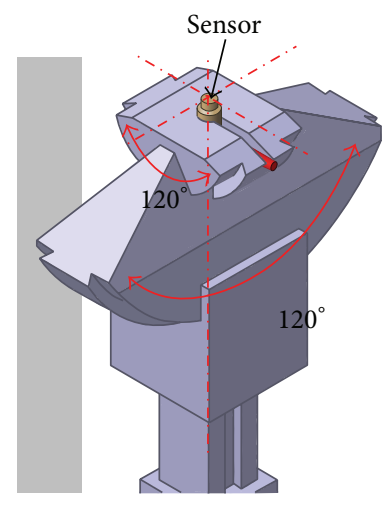

(c)
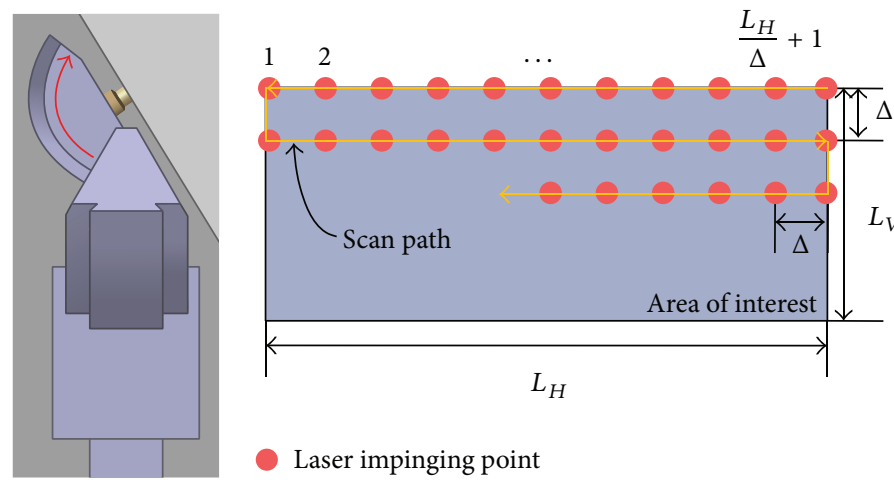

Laser impinging point

FIGURE 3: (a) Schematic diagram of ultrasonic propagation imaging (UPI) system for CRDM assembly inspection, (b) schematic diagram for real-world application of the developed system, (c) sensor contact mechanism, and (d) scan path on the area of interest.

ultrasonic waves using an ultrasonic sensor contacted at one position without reception scanning. The system is designed to automatically visualize the surface cracks when cracks inside the dissimilar metal welding of the RVH have reached the welding surface. This system is based on laser ultrasonic propagation imaging technology [6] and is newly equipped with an advanced platform of multiple damage visualization processing for surface crack visualization. To present a proof-of-concept for the field overhaul application, a fullscale mockup was fabricated and its structural dimensions pertain to the signal-to-noise ratio of the proposed laser ultrasonic technique. Finally, the proposed system is tested for surface crack detection in the dissimilar metal welding of the mockup.

\section{System Configuration}

2.1. Configuration of Dissimilar Weld Crack Visualization System and Experimental Setup. Figure 3(a) shows the system configuration developed in this research for crack detection in CRDM assemblies. Due to the nature of the nuclear reactor vessel, automation of the system is essential to avoid a human directive approach. The RVH head is lifted during the overhaul process of the NPP. During this period, the proposed system shown in Figure 3(b) is inserted below the opened RVH head and makes contact with the ultrasonic sensor on the inner surface of the RVH using its robot arm. High speed laser scanning is then applied around the dissimilar weld using a laser mirror scanner (LMS). 


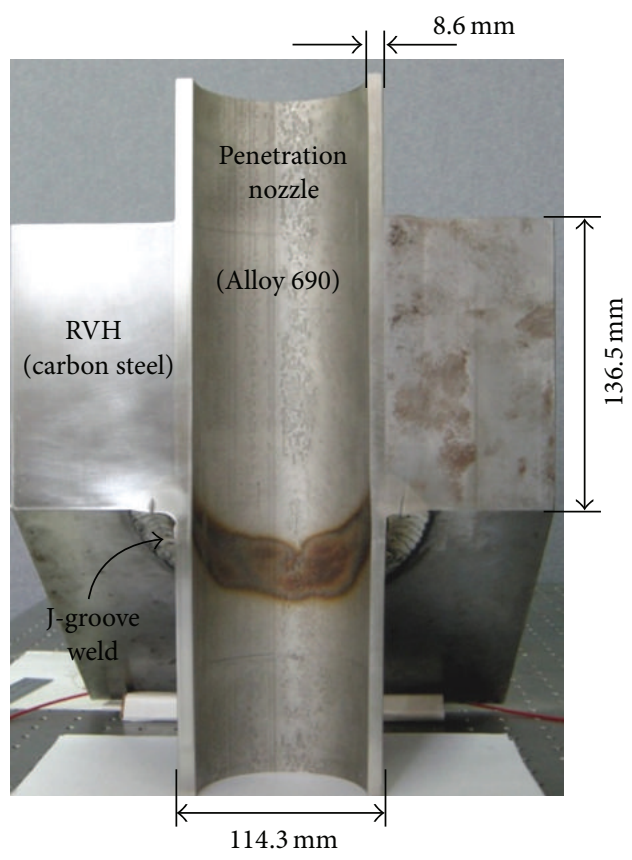

(a)

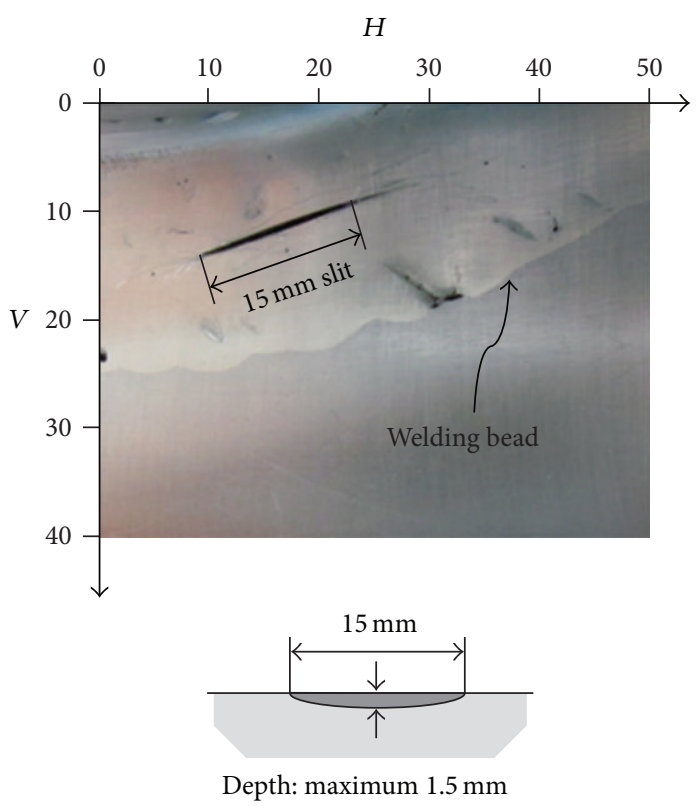

(b)

FIGURE 4: (a) Full-scale mockup of the CRDM assembly and (b) the artificial crack on the dissimilar welding bead.

The Q-switched solid state diode pumped laser (QL) with a wavelength of $532 \mathrm{~nm}$ was used to generate a laser beam pulse with a duration of $8 \mathrm{~ns}$ and the laser mirror scanner (LMS) was used to impinge the laser beam with the same interval on the inner surface of RVH. Impinging of the laser beam in each scanning grid point generated an ultrasonic wave. The LMS was equipped with a pair of laser mirrors for the $532 \mathrm{~nm}$ operating wavelength and the mirrors were fixed on two orthogonal galvanomotors which had $75 \mathrm{rad} / \mathrm{s}$ for maximum angular velocity. Each mirror was able to rotate by \pm 20 degrees from top to bottom or from left to right. The laser pulse was impinged once on each grid point by synchronizing the pulse repetition frequency (PRF) of QL and the movement of galvanomotors. Figure 3(c) shows a sensor contact mechanism installed at the end of a robot arm. An amplifier-integrated PZT sensor with a diameter of $4 \mathrm{~mm}$ and a central frequency of $350 \mathrm{kHz}$ was used. Gel couplant was used on the wear plate of the sensor to increase ultrasonic transmissibility. As shown in Figure 3(d), a laser beam was impinged on each point along the scan path in the area having a width $L_{H}$ and length $L_{V}$ with constant interval $\Delta$. Laser-induced ultrasonic waves were generated on points stimulated by the laser, while the generated ultrasonic waves were detected by the sensor contacted on the target via an inline band-pass filter and saved in the PC for image processing.

Multiple damage visualization algorithms were integrated in this system, such as ultrasonic wave propagation imaging (UWPI) [6], ultrasonic spectral imaging (USI) [6], wavelet transformed ultrasonic propagation imaging (WUPI) [7], and anomalous wave propagation imaging with adjacent wave subtraction (AWPI with AWS, refer as AWS) [8] for damage visualization and localization.
Validation of the proposed system was carried out using the full-scale mockup of the CRDM assembly shown in Figure 4(a). For the carbon steel part for the real RVH with a $136.5 \mathrm{~mm}$ thickness, the alloy 690 pipe for the real penetration nozzle with a $114.3 \mathrm{~mm}$ diameter and an $8.6 \mathrm{~mm}$ thickness was used. A crack with an arc-shaped depth was generated with a length of $15 \mathrm{~mm}$ and maximum depth and width of $1.5 \mathrm{~mm}$ and $0.7 \mathrm{~mm}$, respectively, in the middle on dissimilar metal welds as shown in Figure 4(b). Scanning was fulfilled at a standoff distance of $1 \mathrm{~m}$ for the scan area with $L_{H}=50 \mathrm{~mm}, L_{V}=40 \mathrm{~mm}$, and $\Delta=0.1 \mathrm{~mm}$. The diameter of the impinging beam was $2 \mathrm{~mm}$. The PRF of $\mathrm{QL}$ is equal to the number of impinged points per second. In this study, $200 \mathrm{~Hz}$ PRF was used and thus 200 pulses of laser beam were impinged on 200 points in one second. The scanning time for the interested area was 17 minutes and the damage visualization image processing time was almost in real time. During the experiments, band-pass filtering in a $130 \mathrm{kHz} 150 \mathrm{kHz}$ range was used for narrowband waveform acquisition with high signal to noise ratios, the energy density of QL was $91.45 \mathrm{~mJ} / \mathrm{cm}$, and the ultrasonic sensor was placed on $(40,65) \mathrm{mm}$ against the origin of the scanning area.

\subsection{Basic Ultrasonic Propagation Image Processing. The} UWPI method rearranges 1D time-domain signals in the 3D data structure. As shown in Figure 5(a), one time-domain wave signal is expressed with $N$ sampling points, and $L_{v} / \Delta+1$ signals on one vertical line are saved in one spreadsheet. A $2 \mathrm{D}$ array $N \times\left(L_{v} / \Delta+1\right)$ is created by arranging the signals as shown in Figure 5(d). After generating spreadsheets for every horizontal path of $L_{H} / \Delta+1$, these spreadsheets are 

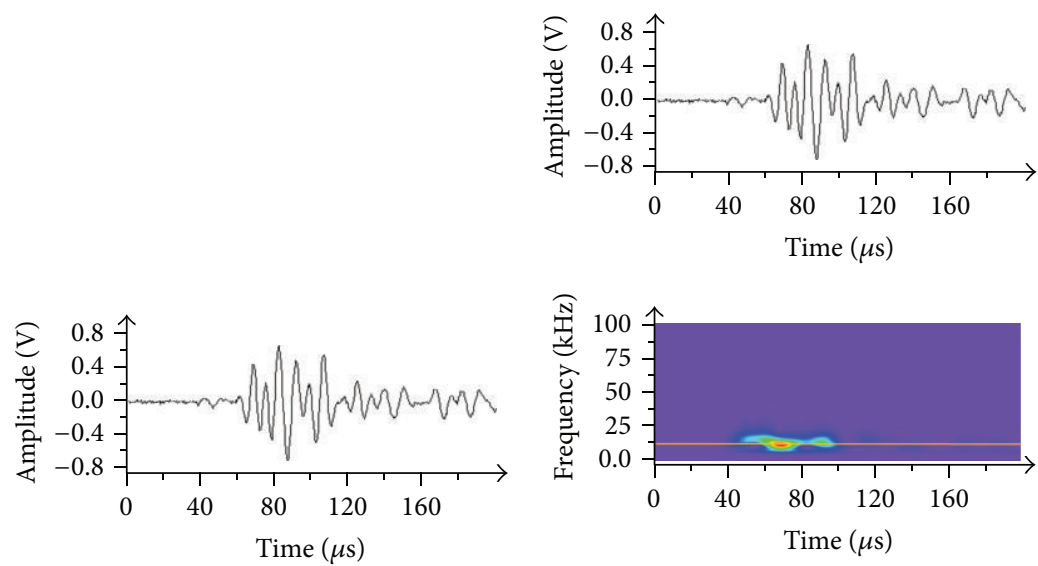

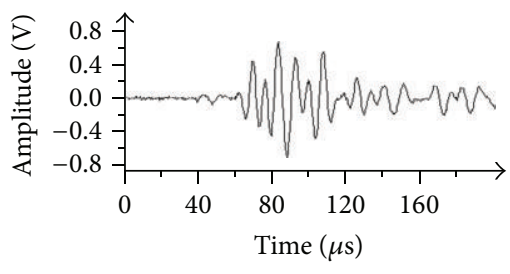

(a)

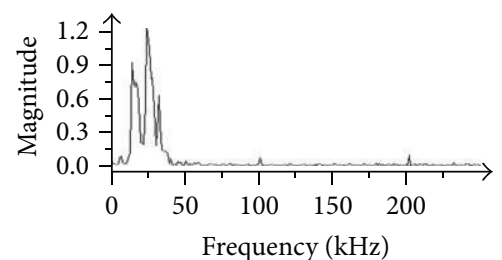

(b)
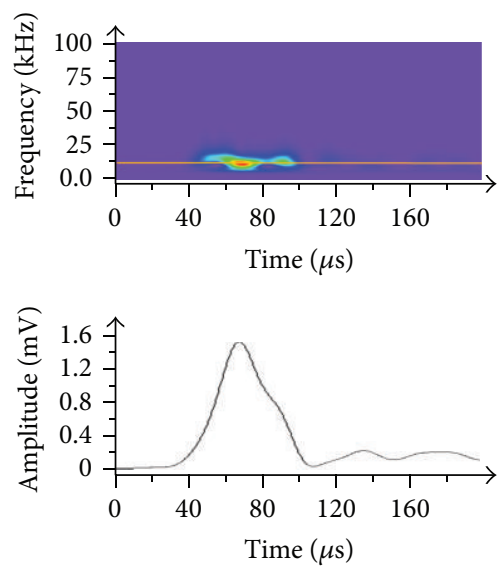

(c)

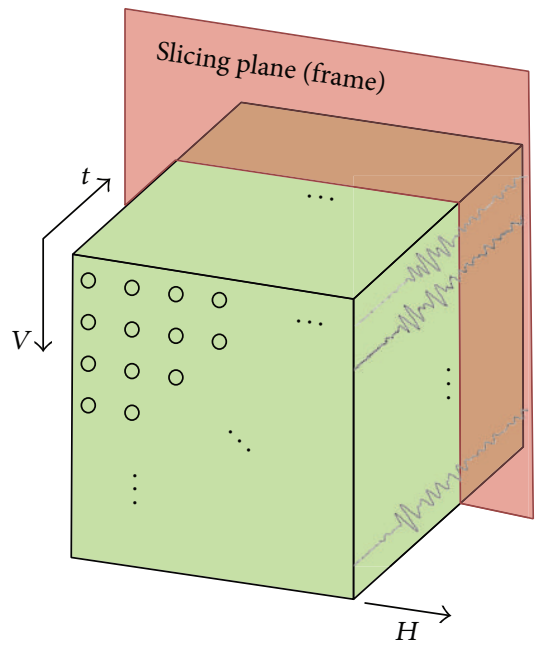

(f)

FIGURE 5: (a) 1D time-domain signal acquired at one of the scanning points, (b) fast Fourier transformed 1D frequency domain signal for USI, (c) wavelet transformed 1D time-domain signal for WUPI, (d) arranged signals along one vertical scan path in one spreadsheet (2D array), (e) arranged spreadsheets along horizontal scan path (3D array), and (f) sliced plane along time axis.

rearranged in a 3D data array structure of $N \times\left(L_{v} / \Delta+\right.$ $1) \times\left(L_{H} / \Delta+1\right)$ as shown in Figure 5(e). The intensity maps expressing the amplitude of signal for each point by color scale are generated by slicing the rotated 3D array along the time axis as shown in Figure 5(f). The intensity maps of $N$ take the role of freeze frames of $N$ in generating the video of wave propagation.

Figure 5(b) shows a 1D frequency-domain signal transformed from the $1 \mathrm{D}$ time-domain signal by applying fast Fourier transform (FFT). In the USI method, these transformed signals are used to generate an ultrasonic spectral video. These signals are arranged in the same way as the
UWPI. Each frame of the USI video expresses the spatial distribution of spectral amplitude with color scale. Here, since the local structural area with damage-induced wave frequency change can be identified in the frame sliced at each frequency, the damaged area is effectively visualized. Also, the frequency component found in the USI that pertains to damage can be utilized in the WUPI. When the wavelettransformed 1D time-domain signal is selected from the wavelet scalogram as presented in Figure 5(c), both the central frequency and the damage-related frequency found can be selected to visualize propagations of dominant wave and damage-related wave, respectively. 


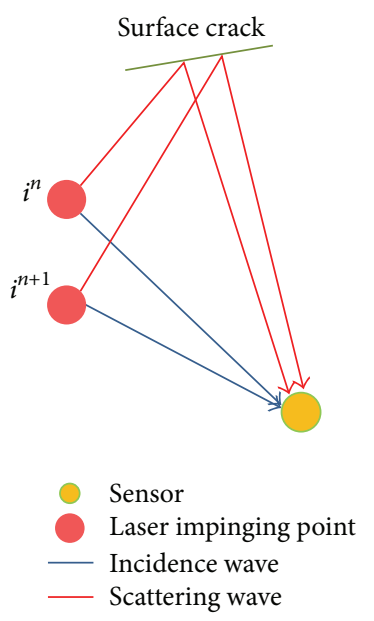

(a)

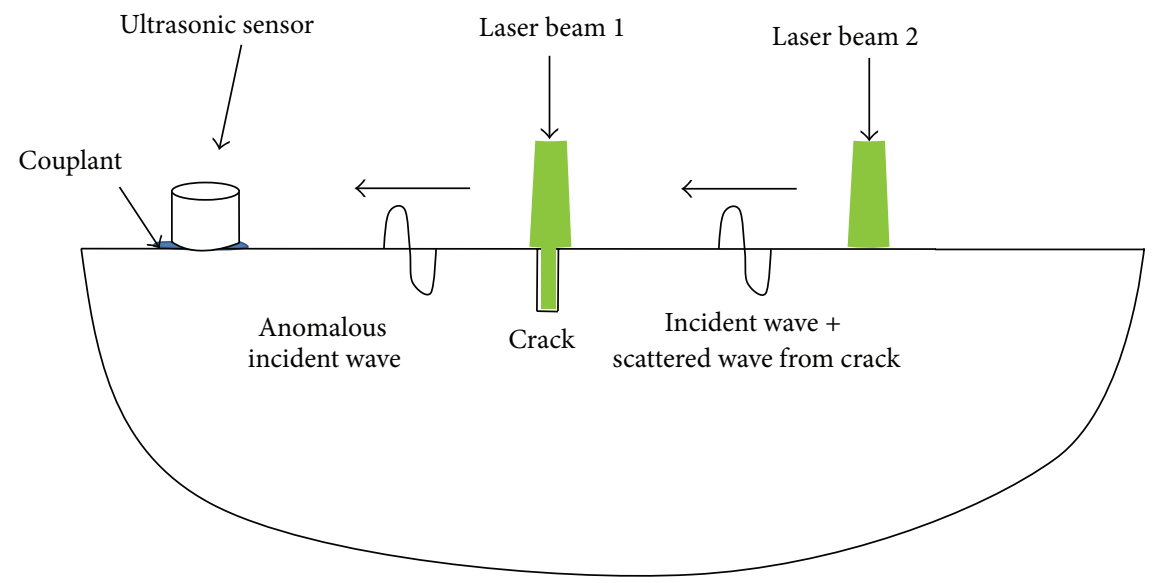

(b)

FIGURE 6: (a) Scattering wave generation mechanism of the beam impingement near the surface crack and (b) anomalous wave generation mechanism of the beam impingement within the surface crack.

The WUPI method generates a wavelet-transformed ultrasonic propagation video by rearranging $1 \mathrm{D}$ wavelettransformed signals with a selected frequency in the same way as that of the UWPI, as presented in Figures 5(d)-5(f).

\subsection{Novel Dual-Directional Anomalous Wave Propagation} Imaging and Crack Orientation. The system developed in this study was equipped with the advanced AWS method developed to be a suitable crack visualization method by modifying the conventional AWS [8] developed for area type damage evaluation. In the case of area type damage such as delamination or debonding, when an ultrasonic wave is generated by a laser beam impinged within the damage area, the confining wave (which is a type of $2 \mathrm{D}$ standing wave which stays in the damaged area) occurs as well as the scattering wave by laser beams impinged around the area damage.

As shown in Figure 6(a), when an ultrasonic wave was generated by a laser beam impinged around the crack $\left(i_{n}\right.$ or $\left.i_{n+1}\right)$, partial ultrasonic wave components bounced off the crack and became scattering waves. In this paper, for the crack as the target which has reached the surface such as the PWSCCs, waves generated near and within the crack as represented in Figure 6(b) should show considerable difference. In general, a wave generated by a laser beam impinged within the damage would have higher amplitude due to the boundary impinging effect and deeper excitation.

Figure 7 (a) shows horizontally adjacent signals acquired at $(40.0,34.2),(40.1,34.2)$, and $(40.2,34.2)$ in an intact region. Similarly, Figure 7(b) shows vertically adjacent signals acquired at $(40.0,34.2),(40.0,34.3)$, and $(40.0,34.4)$. The horizontally or vertically adjacent signals generated on the intact surface show strong similarity to each other, regardless of direction. Figure 7(c) shows signals acquired on the three horizontally adjacent points, $(14.0,12.3),(14.1,12.3)$, and (14.2, 12.3), which are placed on a position close to the artificial crack. These adjacent signals still have strong similarities.
Since the $2 \mathrm{~mm}$ laser beam scanned the surface crack almost along its orientation, the interactions between the laser beam and crack did not considerably differ and the adjacently generated signals thus had strong similarities. On the other hand, the vertically adjacent signals shown in Figure $7(\mathrm{~d})$ differ considerably despite the small interval of $0.1 \mathrm{~mm}$ because the laser beam scanned the surface crack almost perpendicular to its orientation.

As a result, the conventional AWS algorithm, which does not consider the directivity of the crack in selecting the two adjacent signals, might fail to visualize the crack that has an orientation parallel to the processing. Therefore, dual-directional adjacent wave subtraction (DAWS) which processes both directional adjacent signals is proposed in this paper.

The AWS algorithm includes a signal processing step which allows the matching of two signals that have different arrival times. Arrival time matching is accomplished by shifting the time axis of the second signal in scanning the two signals, while the number of sampling points to be shifted is determined when the subtracted signal, $s(N)_{i}-s(N \pm l)_{i+1}$, has minimum root mean square (RMS) value

$$
s_{\mathrm{RMS}}=\sqrt{\frac{\sum_{k+l_{\max }}^{N-l_{\max }}\left[s(N)_{i}-s(N \pm l)_{i+1}\right]}{N-2 l_{\max }}},
$$

where $l$ is the amount of the shifting, and $T$ and $N$ denote the sampling interval and the number of data points during the signal acquisition, respectively. The shifted time could be expressed as $l(T / N)$ (sec.). The first signal $s(N)_{i}$ and second signal $s(N)_{i+1}$ are considered to be matched when the calculated $s_{\mathrm{RMS}}$ in $l_{\max }(T / N)<t<T-l_{\max }(T / N)$ is minimum. Therefore, the two adjacent waves obtained in an intact area are similar, even before the time point adjustment is made to minimize their difference; however, two adjacent 


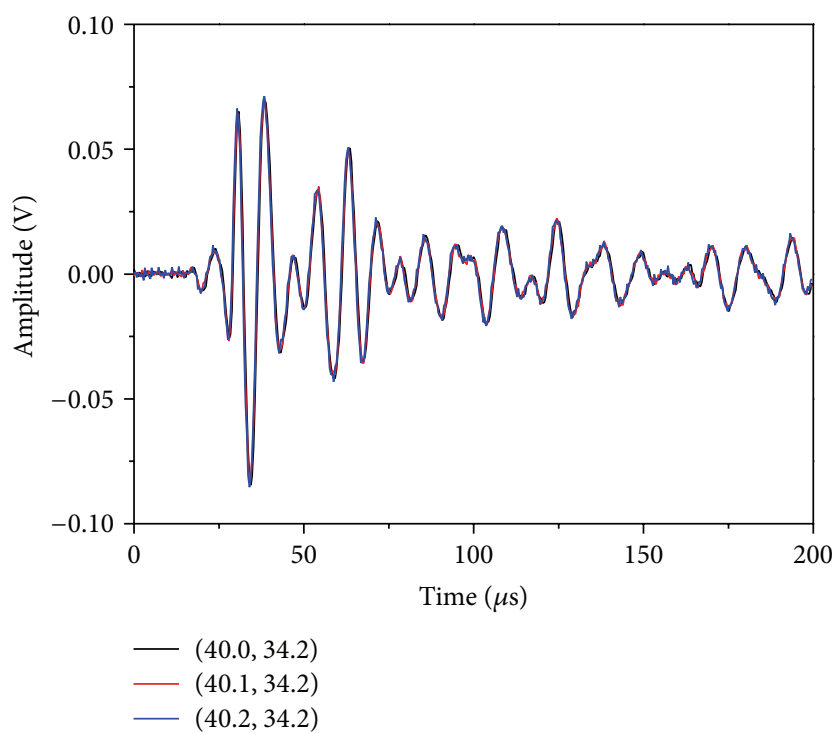

(a)

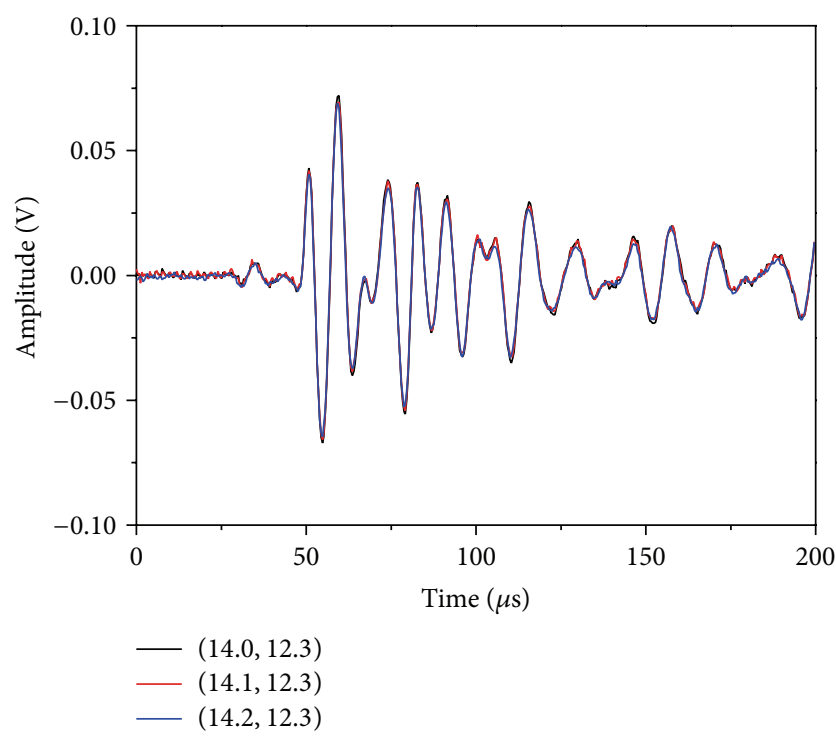

(c)

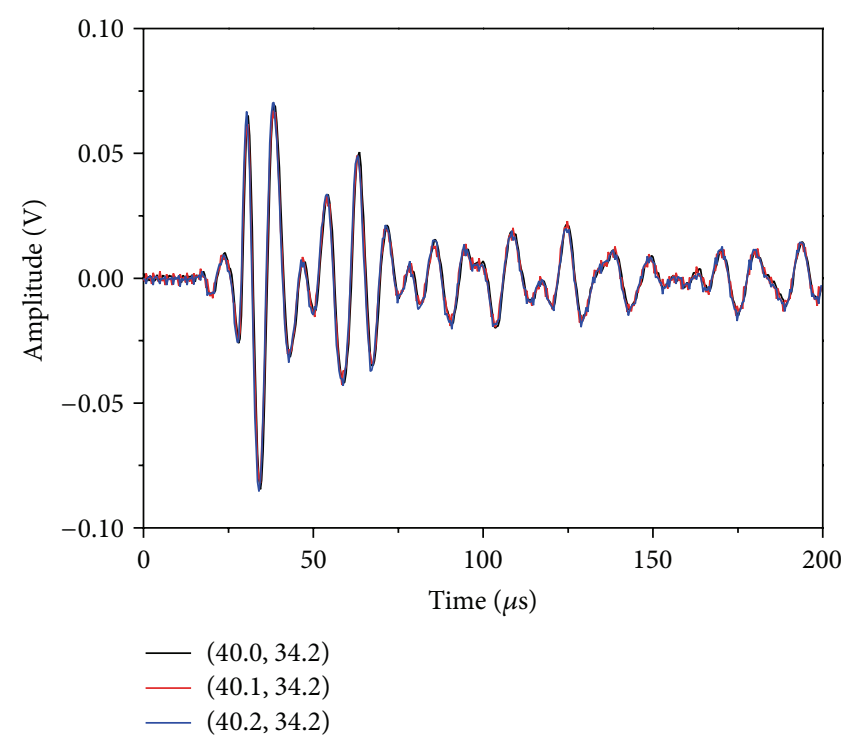

(b)

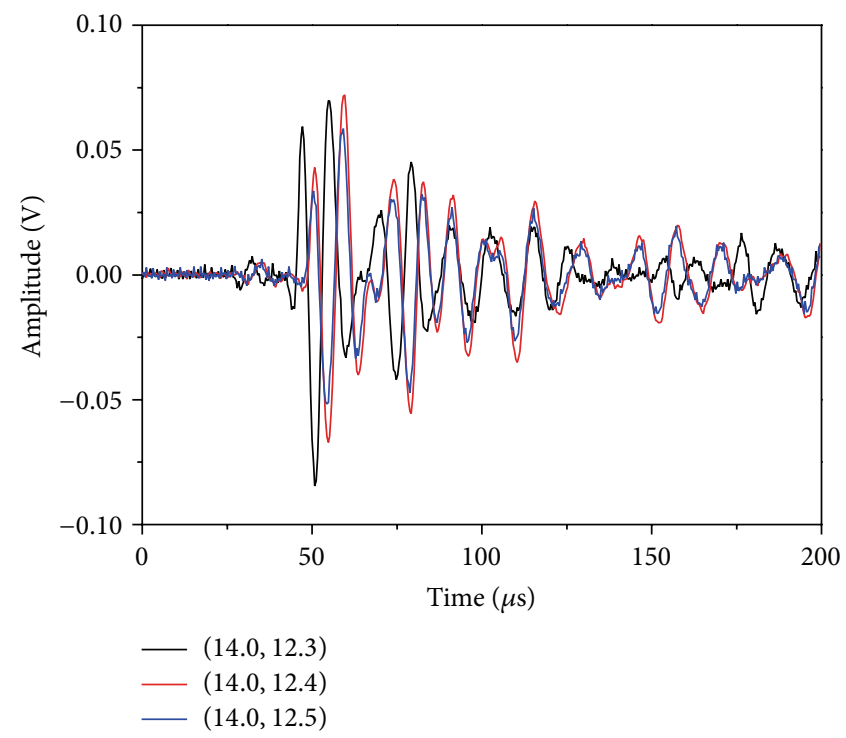

(d)

FIgURE 7: Similarities of three adjacent signals along (a) $H$-axis, (b) $V$-axis at an intact surface position, (c) $H$-axis, and (d) $V$-axis at the surface crack position.

waves obtained in a damaged area differ even after the time point adjustment and thus the subtracted signal still contains a visible residual component. The nature of the residual wave is dependent on the damage types which can cause a residual of incidence wave, scattering wave, and/or confining wave.

Figure 8(a) shows the result of horizontally adjacent wave subtraction using the first signal at $(14.0,12.3)$ and second signal at $(14.1,12.3)$ on the surface crack. The subtracted signal shows low residual of incidence wave because of the high similarity of the horizontally adjacent waves and the parallel crack orientation. Thus, the AWS with horizontally adjacent wave subtractions failed to increase visibility of anomalous wave components, which can be clarified in Figure 9(d). On the other hand, Figure 8(b) shows the result of vertically adjacent wave subtraction using the first signal at $(14.0,12.3)$ and second signal at $(14.0,12.4)$, where it shows high residual of incidence wave with high amplitude even though the signals have the same interval as that of the signals of Figure $8(\mathrm{a})$. The result of vertically adjacent wave subtraction shown in Figure 8(c) was calculated by using signals at $(40.0,34.2)$ and $(40.0,34.3)$, which are independent of the surface crack position. Even if it was also processed with vertically adjacent waves such as shown in Figure $8(\mathrm{~b})$, the residual was completely removed by the time point adjustment and subtraction. Consequently, the crack orientation is unknown in real-world applications; DAWS thus needs to be used to not overlook the random directional cracks. 

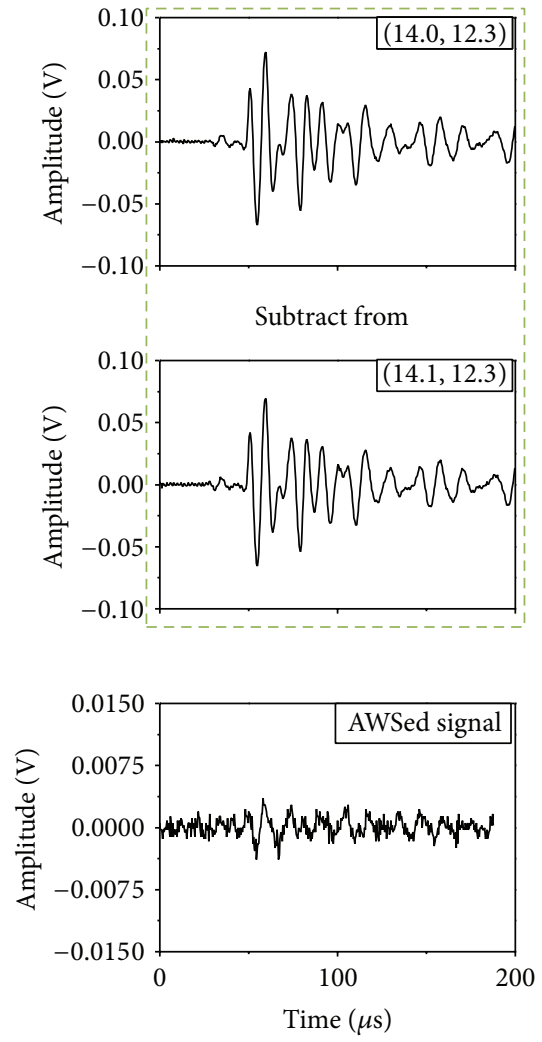

(a)

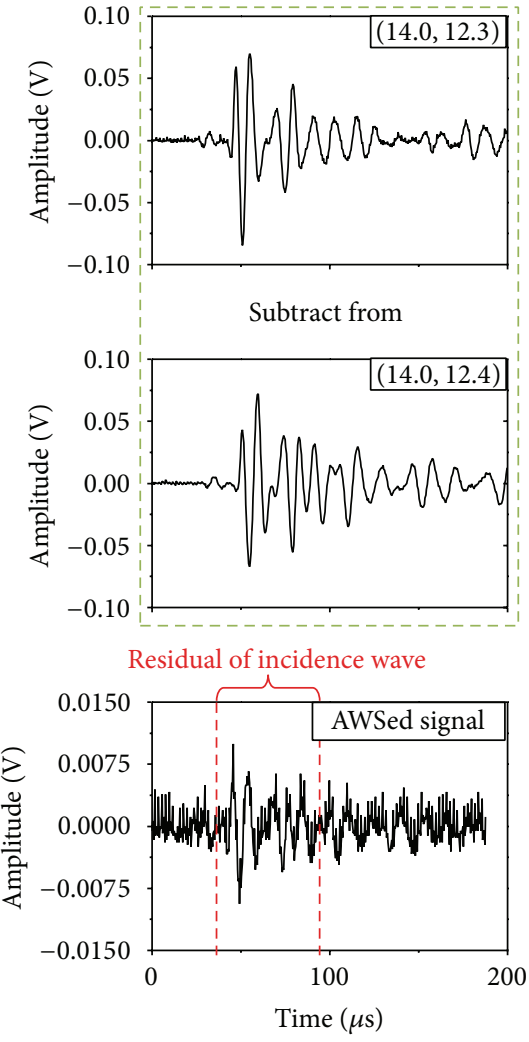

(b)
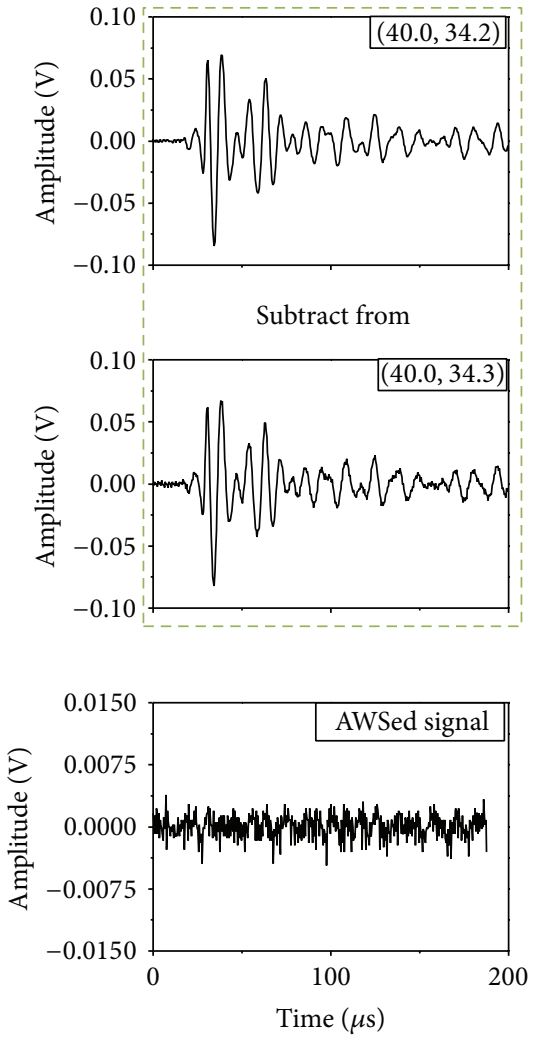

(c)

FIGURE 8: Adjacent wave subtraction process using signals obtained at (a) (14.0, 12.3) and (14.1, 12.3), horizontal AWS; (b) (14.0, 12.3), (14.0, 12.4), and vertical AWS on the surface crack; and (c) $(40.0,34.2),(40.0,34.3)$, and vertical AWS on the intact area.

\section{Experimental Results}

Figure 9(a) shows the freeze frame at $29.4 \mu$ s of the UWPI video result, which is the moment when the propagating wave interacts with the crack as presented in Figure 4(b). Since the thickness of the CRDM assembly is at the limit for still being considered a semi-infinite structure, the Rayleigh surface wave [9] is dominative the longitudinal waves and the Rayleigh waves are observed in the UWPI movie. The position and shape of the crack were able to be detected from the length of the collapsed wave front and the length of the high amplitude pattern along the surface crack line. The main frequency component induced by damage could be distinguished by the USI. As shown in Figure 9(b), the freeze frame at $140 \mathrm{kHz}$ clearly isolates the shape and location of the surface crack on the dissimilar weld. The same frequency component was also used to generate the WUPI video result as shown in Figure 9(c). The freeze frame of the WUPI video presents an ultrasonic wave component corresponding to only the narrowband of $140 \mathrm{kHz}$. In the case of UWPI, since all frequency components are mixed in one signal, a very complex propagation pattern is shown. On the other hand, WUPI shows a simpler propagation pattern because it uses only one selected frequency component; thus, it can extract a signal component related to damage with greater visibility. However, a number of steps are required to find the changed frequency components and if the damage did not cause any change in the frequency components, WUPI might fail to visualize this damage.

The DAWS algorithm described in Section 2.3 was applied and the freeze frame for the horizontally processed AWS video result is shown in Figure 9(d). The damage could not be visualized in the horizontal direction because the directivity of the surface crack is also approximately horizontal. A small difference was induced between the two adjacent signals; thus considerably less residual incidence waves remained on the subtracted signals. On the other hand, as shown in Figure 9(e), the vertically processed AWS video result visualized the artificial crack exclusively with high contrast. It provided much better visibility of the crack by suppressing most incidence waves not related to the damage and was also able to visualize the complex welding bead in the CRDM assembly.

The crack lengths estimated in the respective imaging methods are indicated in the freeze frames and show good agreement with the real crack length of $15 \mathrm{~mm}$. Since this multiple image processing platform cross-checks the damaged or nondamaged area from the random noisy area, inspectors can make more reliable decisions about the damage. 


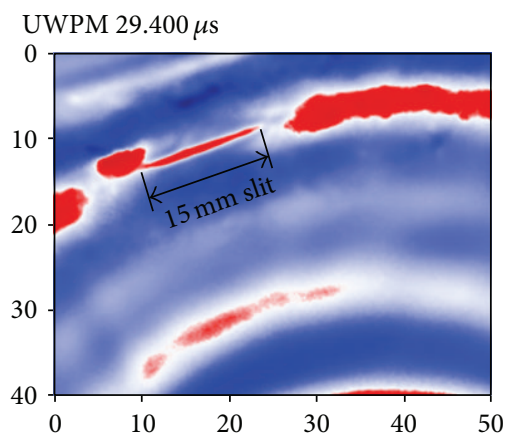

(a)

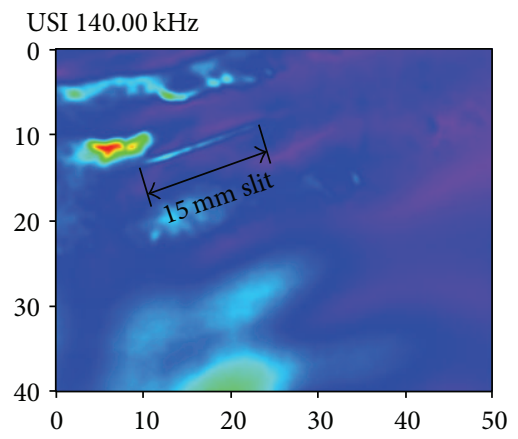

(b)

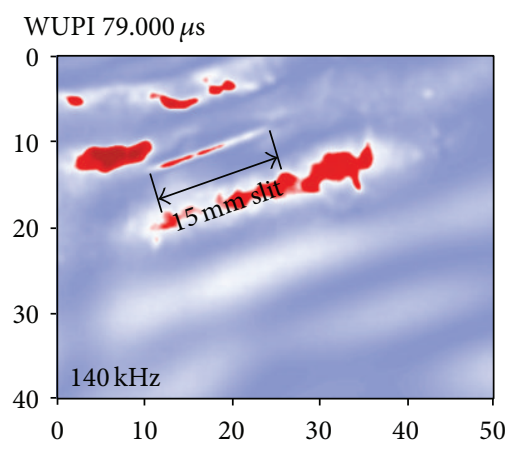

(c)

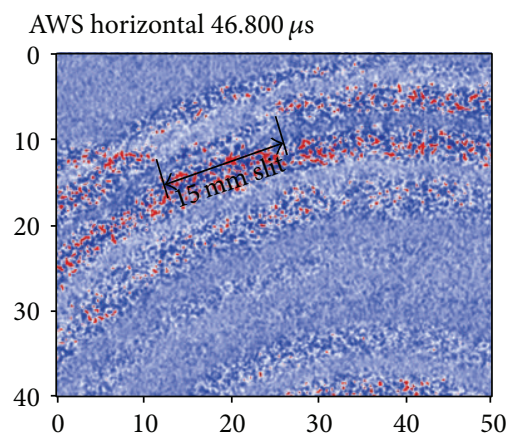

(d)

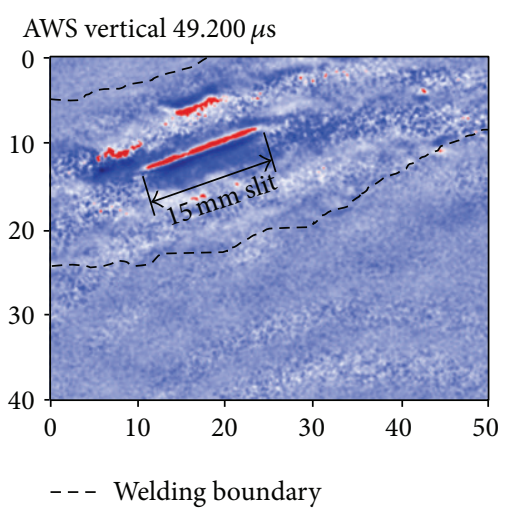

(e)

FIgURE 9: Multiple damage visualization results for a $15 \mathrm{~mm}$ surface crack in the welding, freeze frames at (a) $29.4 \mu \mathrm{s}$ of the UWPI video, (b) $140 \mathrm{kHz}$ of the USI video, (c) $79 \mu \mathrm{s}$ and $140 \mathrm{kHz}$ of the WUPI video, (d) $46.8 \mu \mathrm{s}$ of the horizontal AWS video, and (e) $49.2 \mu \mathrm{s}$ of the vertical AWS video.

\section{Conclusion}

A novel laser ultrasonic crack visualization system was designed and fabricated to detect and evaluate PWSCCs on dissimilar welds between penetration nozzles and an RVH of a CRDM assembly based on the ultrasonic propagation imaging technology. A multiple-damage visualization platform consisting of the four algorithms, UWPI, USI, WUPI, and DAWS, was equipped in the proposed system.

The $\mathrm{RVH}$ is lifted during the regular overhaul process of the NPP. During this period, the proposed system is operated and its robot arm contacts the ultrasonic sensor on the inner surface of the RVH and high speed laser scanning is performed around the dissimilar weld. For the proof-ofconcept, a full-scale mockup for the CRDM assembly was fabricated and a $15 \mathrm{~mm}$ artificial surface crack was generated in the welding bead. The system feasibility has been proved by visualizing the crack length exactly. In particular, the newly developed DAWS was able to visualize any crack regardless of its orientation.

The dense scanning grid with $0.1 \mathrm{~mm}$ scanning interval was realized for exhaustive inspection of the complex welding area of $40 \mathrm{~mm} \times 50 \mathrm{~mm}$. The scanning time was only 17 minutes and damage visualization image processing was completed in real time, thereby minimizing the economic loss induced by the interruption of plant operation.

\section{Conflict of Interests}

The authors declare that there is no conflict of interests regarding the publication of this paper.

\section{Acknowledgments}

This research was supported by the Basic Science Research Program (2011-0010489) and the Leading Foreign Research Institute Recruitment Program through the National Research Foundation of Korea funded by the Ministry of Education, Science and Technology (2011-0030065).

\section{References}

[1] Nuclear Regulatory Commission, Davis-Besse Reactor Pressure Vessel Head Degradation: Overview, Lessons Learned, and NRC Actions Based on Lessons Learned (NUREG/BR-0353, Revision 1), 2008.

[2] R. Schrauder, "Lessons learned-reactor pressure vessel degradation: material degradation and related managerial issues at nuclear power plants," in Proceedings of the Technical Meeting, pp. 33-38, International Atomic Energy Agency, Vienna, Austria, 2005.

[3] H. Ito and M. Kameyama, "Trend of PWSCC in nuclear reactor vessel head penetrations," E-Journal of Advanced Maintenance, vol. 1, no. 1, pp. 1-7, 2009. 
[4] S. E. Cumblidge, S. R. Doctor, G. J. Schuster et al., "NDE and DE of PWSCC found in the j-groove weld of a removed-fromservice control rod drive mechanism," in Proceedings of the 6th International Conference on NDE in Relation to Structural Integrity for Nuclear and Pressurized Components, pp. 723-733, Budapest, Hungary, 2007.

[5] D. S. Kerr and R. B. Alers, "Method and system for nondestructive inspection of components," Tech. Rep. U.S. Patent No. 2004/0091076 Al, 2004.

[6] J.-R. Lee, H. Jeong, C. C. Ciang, D.-J. Yoon, and S.-S. Lee, "Application of ultrasonic wave propagation imaging method to automatic damage visualization of nuclear power plant pipeline," Nuclear Engineering and Design, vol. 240, no. 10, pp. 3513-3520, 2010.

[7] J.-R. Lee, C. C. Chia, H. J. Shin, C.-Y. Park, and D. J. Yoon, "Laser ultrasonic propagation imaging method in the frequency domain based on wavelet transformation," Optics and Lasers in Engineering, vol. 49, no. 1, pp. 167-175, 2011.

[8] J.-R. Lee, C. C. Chia, C.-Y. Park, and H. Jeong, "Laser ultrasonic anomalous wave propagation imaging method with adjacent wave subtraction: algorithm," Optics and Laser Technology, vol. 44, no. 5, pp. 1507-1515, 2012.

[9] J. L. Rose, Ultrasonic Waves in Solid Media, Cambridge University Press, New York, NY, USA, 1999.

[10] D. Schlader, "The evolution of inspection and repair approaches for reactor vessel head penetrations," in Proceedings of the Conference on Vessel Penetration Inspection, Crack Growth and Repair, vol. 1, pp. 81-148, Gaithersburg, Md, USA, 2003. 

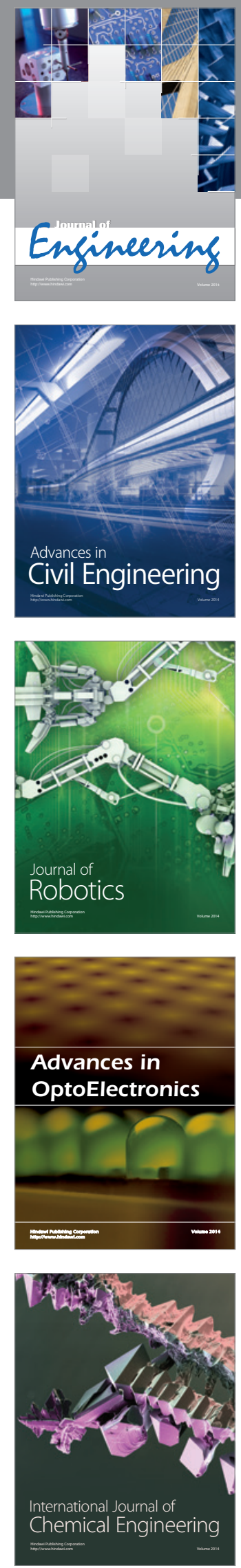

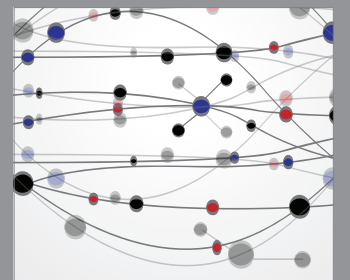

The Scientific World Journal
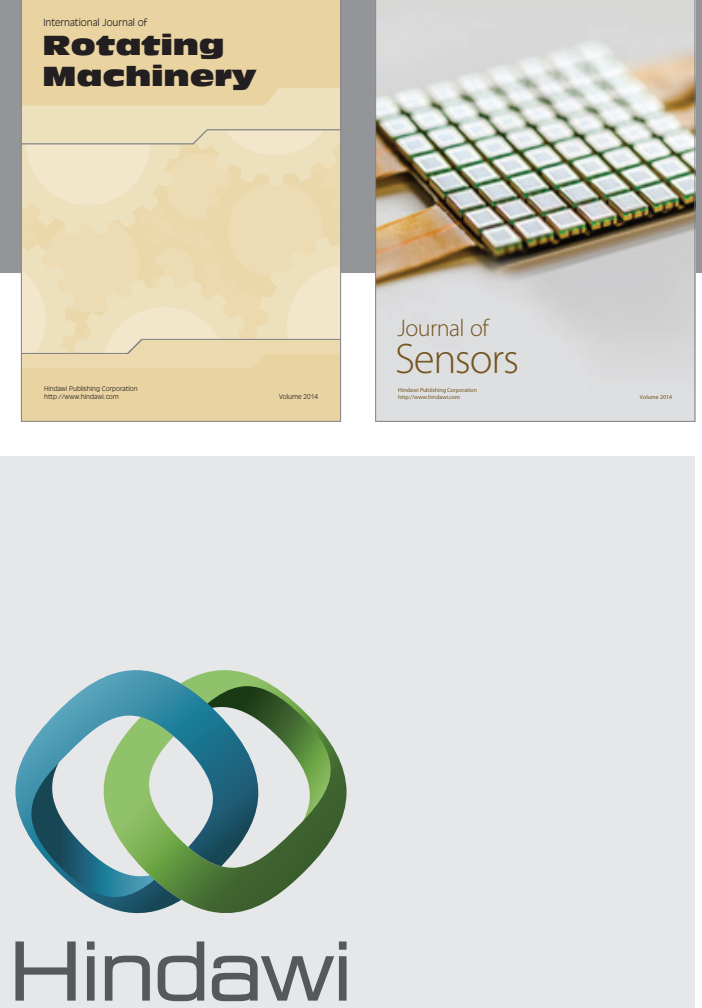

Submit your manuscripts at http://www.hindawi.com
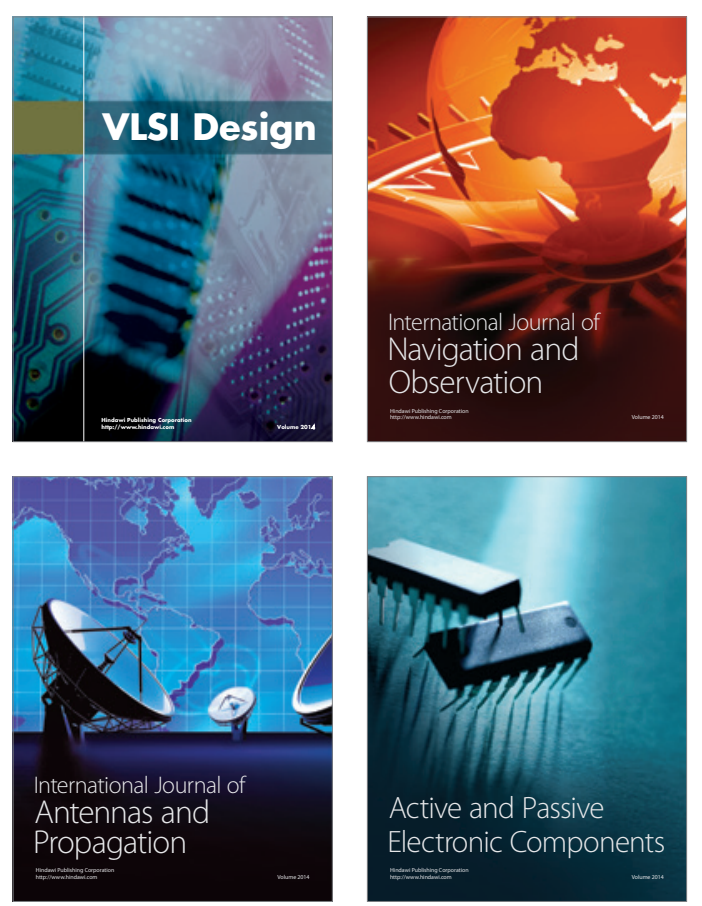
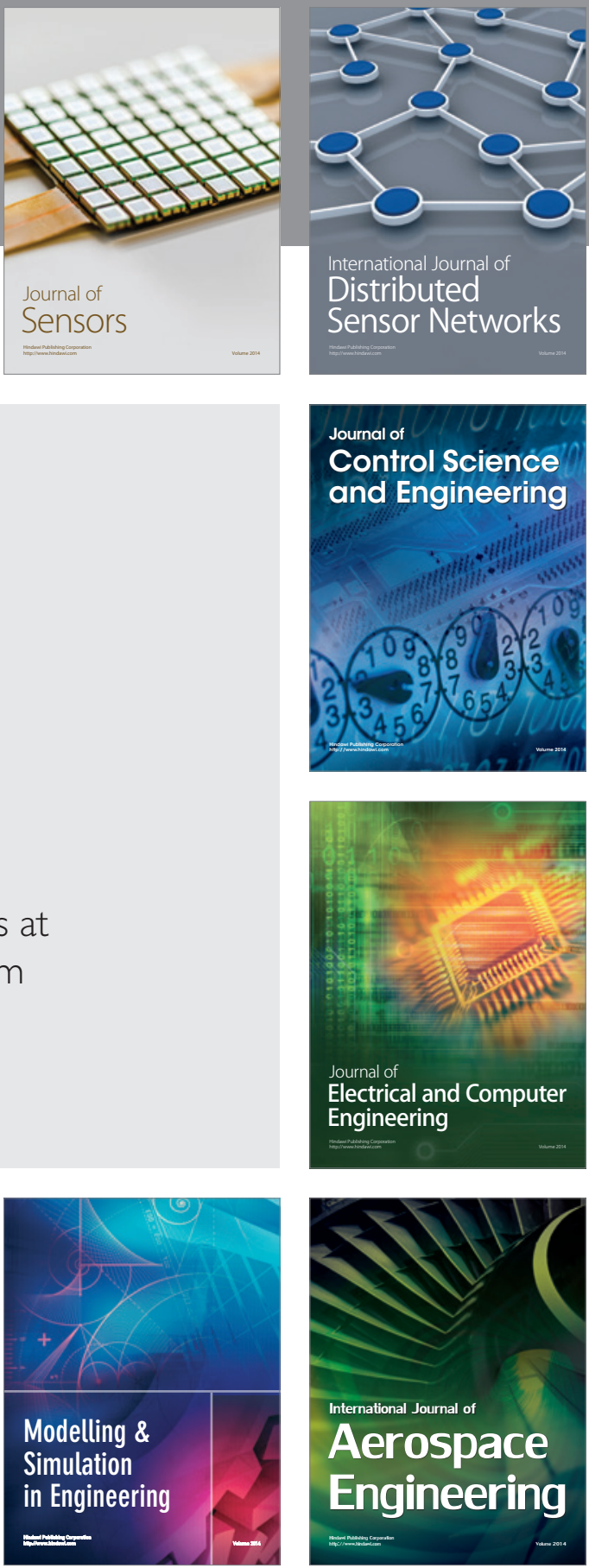

Journal of

Control Science

and Engineering
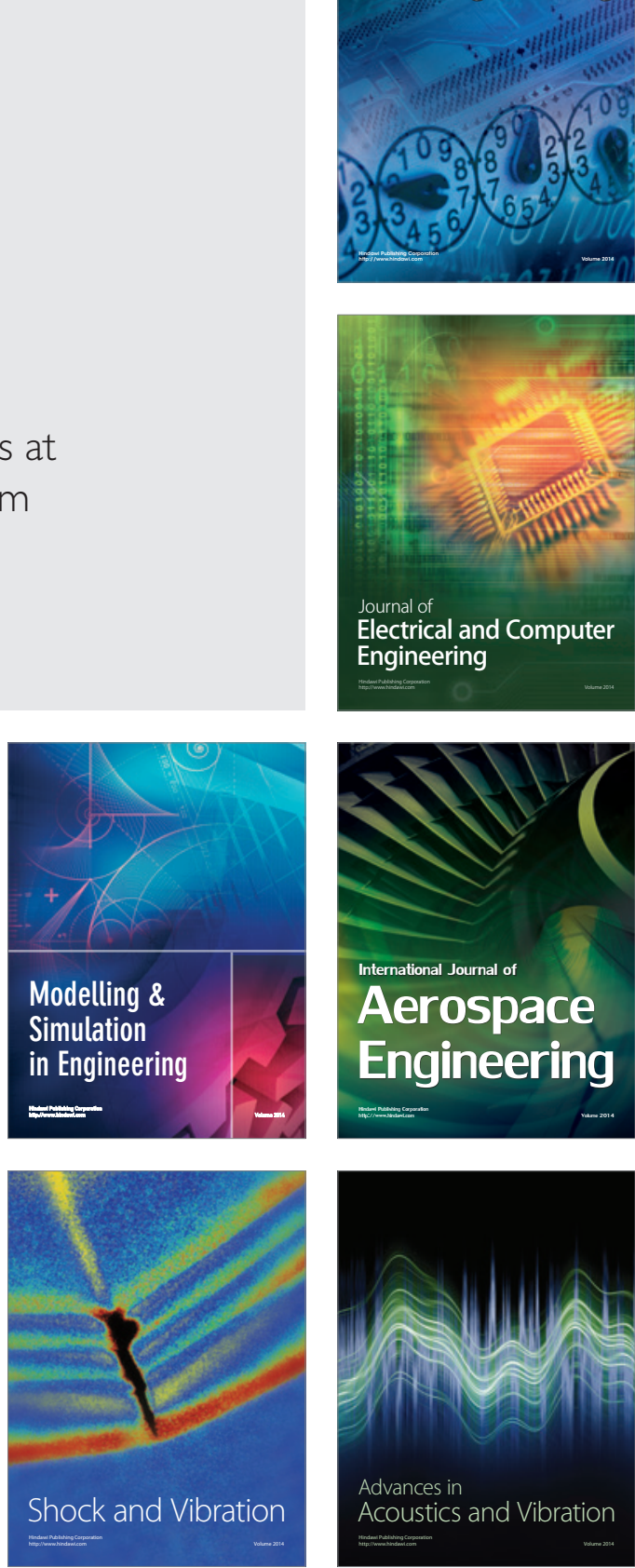